\title{
La paradiplomacia universitaria, las transformaciones de las universidades ante el COVID 191
}

\author{
University paradiplomacy, the transformations of universities in the face of COVID 19 \\ María Gabriela, Zapata-Morán²; Jorge Hipólito, Berlanga-Ramírez ${ }^{3}$, Hugo, Salazar-Mata ${ }^{4}$
}

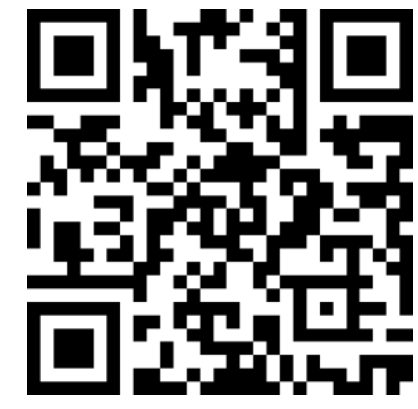

Fecha de recibido: $20-12-2020$

Fecha de aceptado: 03-03-2021

\section{(cc) BY-NC-ND}

Esta obra está bajo una licencia de Creative Commons Reconocimiento-NoComercialSinObraDerivada 4.0 Internacional

\begin{abstract}
RESUMEN
El modelo de universidad pública y privada en México ha venido a transformarse con la llegada de la pandemia por el COVID-19. Las nuevas estrategias de la paradiplomacia universitaria crean un nuevo sistema universitario capaz de integrar estrategias digitales y relaciones interinstitucionales a distancia. El objetivo de este artículo es recopilar las diferentes estrategias que se implementan para llevar a cabo acciones paradiplomáticas en las universidades y el impacto que éstas tienen en la atracción de estudiantes extranjeros que ingresan a un Posgrado a la UANL. En un estudio realizado antes y durante la pandemia se realizó un instrumento cuantitativo para la recolección de datos a través de encuestas aplicadas a estudiantes extranjeros. El presente artículo es producto de una revisión documental y literaria en donde se encontró que el contraste de resultados indicó una transformación en la empresa universitaria y su internacionalización durante la pandemia. Los nuevos modelos universitarios traen consigo nuevas estrategias de paradiplomacia que permitan comprender la dimensión internacional de las universidades como actores locales.
\end{abstract}

Palabras claves: COVID-19, Internacionalización de la educación superior, Paradiplomacia universitaria.

\begin{abstract}
The public and private university model in Mexico has been transformed with the arrival of the COVID-19 pandemic. The new strategies of university paradiplomacy create a new university system capable of integrating digital strategies and inter-institutional relations at a distance. The objective of this article is to compile the different strategies that are implemented to carry out paradiplomatic actions in universities and the impact they have on attracting foreign students who enter a Postgraduate degree at UANL. In a study carried out before and during the pandemic, a quantitative instrument was used to collect data through surveys applied to foreign students. This article is the product of a documentary and literary review where it was found that the contrast of results indicated a transformation in the university company and its internationalization during the pandemic. The new university models bring with them new paradiplomacy strategies that allow us to understand the international dimension of universities as local actors.
\end{abstract}

Keywords: COVID-19, Internationalization of higher education, University paradiplomacy.

Cómo referenciar este artículo:

Zapata-Morán., M., G., Berlanga-Ramírez, J., H., \& Salazar-Mata, H. (2021). La paradiplomacia universitaria, las transformaciones de las universidades ante el COVID 19. Revista Política Globalidad y Ciudadanía, 7(14), 233-253. https://doi.org/10.29105/pgc7.14-11

\footnotetext{
${ }^{1}$ Este artículo es producto del proyecto "Las estrategias de Paradiplomacia Universitaria en la atracción de estudiantes extranjeros de Posgrado de la UANL", financiado por el CONACYT. Iniciado en 2018 y finalizado en 2021.

${ }^{2}$ Doctora en Relaciones Internacionales, Negocios y Diplomacia por la Universidad Autónoma de Nuevo León, Profesora en la Facultad de Ciencias Políticas y Relaciones Internacionales de la Universidad Autónoma de Nuevo León, Monterrey. Email: gabrielazapmor15@gmail.com. Orcid: https://orcid.org/0000-0002-4386-084X.

${ }^{3}$ Doctor en gerencia pública y política social por la Universidad de Baja California, Profesor titular en la Facultad de Ciencias Políticas y Relaciones Internacionales de la Universidad Autónoma de Nuevo León, Monterrey. Email: polo_rmz@ hotmail.com Orcid: https://orcid.org/0000-0002-4509-6830.

${ }^{4}$ Doctor en Filosofía con orientación a la Gobernabilidad, Universidad Autónoma de Nuevo León, Monterrey. Profesora en la Facultad de Ciencias Políticas y Relaciones Internacionales de la Universidad Autónoma de Nuevo León, Monterrey. Email: hugosalazarm@gmail.com. Orcid: https://orcid.org/0000-0002-6254-3436.
} 


\section{1.- INTRODUCCIÓN}

En un primer aparatado se explica a través de las palabras de Zeraoui que la Paradiplomacia, a diferencia de la diplomacia, que es exclusiva del poder político federal y no de entidades privadas, la paradiplomacia es una actividad reservada a los gobiernos intermedios, provincias o municipalidades y no a las instituciones privadas. La primera vez que apareció el termino fue en la década de los 80 `s y los pioneros fueron Duchaceck y Soldatos desde entonces ha evolucionado a través del tiempo en diferentes concepciones. Además de incluir nuevos actores que no son necesariamente gobiernos locales o subnacionales si no que se integran nuevos participantes en el sistema internacional como las universidades o las escuelas de posgrado.

Es por eso por lo que en los últimos años el mundo ha visto una creciente participación de los gobiernos regionales en el ámbito internacional, un fenómeno a veces conocido como paradiplomacia. Las razones radican tanto en cambios a nivel del sistema estatal e internacional, y en el plano político, así como el desarrollo económico dentro de las propias regiones (Farías, 2014).

En el estudio realizado por Velázquez (2007) menciona que el incremento de las actividades paradiplomáticas se debe a que existe una mayor interdependencia económica y una creciente globalización. En este sentido, la participación de los actores no centrales en los asuntos internacionales se debe a la globalización que ha limitado paulatinamente la capacidad de los Estados centrales de satisfacer las necesidades de las regiones.

En cuanto al porqué de la paradiplomacia universitaria y su concepción para el estudio de Ruiz Gutiérrez se define más bien la participación de diferentes actores y no solo limitados a los gobiernos no centrales, abarcando todos los actores que la ejecutan (empresas, universidades, organismos internacionales, etc.), busca responder a los cambios y necesidades globales y, dependiendo del rubro de cada actor, estos trabajan para ese fin común de integración y desarrollo; desarrollo del sector económico en el caso de las empresas, sector académico y cultural si hablamos de actores educativos y estos en conjunto a nivel macro trabajan por un desarrollo regional (Ruiz-Gutiérrez, 2017).

Las universidades han recibido el impacto de la globalización, su participación en el escenario internacional depende en gran medida de sus estrategias, motivaciones y capacidades. Estas acciones 
tienen un papel importante en el cambio institucional al interior de las universidades; sin embargo, también impactan en la región en donde se desarrollan.

Los estudiantes de Posgrado internacionales buscan cada vez más la movilidad como referencia de calidad en sus estudios, una respuesta a la tendencia de la globalización. Las universidades tendrán que adaptarse a los cambios de este fenómeno internacional.

\section{La paradiplomacia universitaria}

En la paradiplomacia se toma entonces el sentido vital a través de la internacionalización de las universidades, puesto que estas relaciones internacionales paralelas del profesor, investigador y de la propia universidad fortalecen sus capacidades para el futuro. El papel que ejercen las universidades que se comportan como actores no tradicionales, es clave para fomentar el desarrollo socioeconómico de las regiones a las que pertenecen.

Por otro lado, la Paradiplomacia ejerce un rol fundamental en la atracción de estudiantes extranjeros, pues sus estrategias de acción son usualmente dirigidas hacia ciudadanos globales. De acuerdo a la Organización de las Naciones Unidas(ONU) la ciudadanía global se entiende como un tipo de ciudadano que trasciende el espacio de lo nacional, se sustrae de un ámbito identitario o territorial específico, y se integra a una ética global en constante desarrollo (ONU, 2016).

\section{Tabla 1.}

Total, de estudiantes extranjeros matriculados.

\begin{tabular}{llrrr}
\hline Institución/periodo & $\begin{array}{l}\text { Total, } \\
\text { alumnos } \\
\text { extranjeros } \\
\text { matriculados }\end{array}$ & $\begin{array}{l}\text { Total, de } \\
\text { alumnos } \\
\text { extranjeros } \\
\text { matriculados }\end{array}$ & $\begin{array}{l}\text { Total, } \\
\text { alumnos } \\
\text { extranjeros } \\
\text { matriculados }\end{array}$ & $\begin{array}{l}\text { Total, } \\
\text { alumnos extranjeros }\end{array}$ \\
& $2014-2015$ & $2015-2016$ & $2016-2017$ & matriculados \\
UANL & 1097 & 901 & 789 & 588 \\
ITESM & 5107 & 2381 & 5060 & 5137 \\
UDEM & 2338 & 1694 & 1853 & 523 \\
\hline
\end{tabular}

Fuente: Tomado de Zapata-Morán con datos de las ANUIES, 2019.

En este sentido los Posgrados como especialización y ampliación del conocimiento tienen un papel importante en la formación de los ciudadanos globales; aunque por un lado se presenta un 
Zapata-Morán., M., G., Berlanga-Ramírez, J., H., \& Salazar-Mata, H.

fenómeno sin precedentes en cuanto a la cantidad de estudiantes que deciden continuar sus estudios de Posgrado en el Estado de Nuevo León, también es un hecho que no son los suficientes para la infraestructura y capacidad de la misma. En el estudio realizado por Zapata Morán (2021), se hace una relación entre la cantidad de estudiantes extranjeros que estudian en universidades del área metropolitana de Nuevo León y la cantidad de estudiantes de movilidad nacional que estudian en el extranjero.

A través de esta investigación se pretende estudiar el máximo potencial a explotar de los posgrados, como receptores de estudiantes extranjeros, para fomentar la movilidad estudiantil y la cooperación internacional. Diversos estudios realizados por Sebastián (2005), Melissen (2005), Abdolalizadeh (2014), Albatch \& De wit (2020) se han enfocado en analizar la relación existente entre la estructura de los sistemas educativos y los estudiantes. Algunos concluyeron que los sistemas escolares menos integrales, que dividieron a los estudiantes ósea que no promovían la multiculturalidad, son causantes de agrandar las desigualdades individuales entre los estudiantes y maestros.

Es importante evaluar las estrategias llevadas a cabo por las Universidades estatales y este caso las llevadas a cabo por las escuelas de posgrado pues los estudiantes de este nivel buscan con más esfuerzo crear redes de colaboración con sus pares en el extranjero. Por lo que resulta importante analizarlo, pero no solo desde la perspectiva de los expertos, sino también de los estudiantes saber cuáles son las necesidades y los retos que los universitarios que se integran a un contexto internacional enfrentan y como atendiendo esas necesidades, las universidades encuentran áreas de oportunidad.

La medición de la calidad en los servicios de movilidad prestados en las universidades, en este caso la Universidad Autónoma de Nuevo León, requiere de una búsqueda de más definiciones y categorías, pues para llegar a la estandarización internacional hay temas que están estrechamente vinculados a la Paradiplomacia universitaria.

En la era de la globalización las universidades han demostrado a través de su estabilidad institucional, ser agentes de larga duración parecida incluso a la de los estados nacionales. Esto en gran medida por el papel que tienen en la formación de clases dirigentes y de técnicos y profesionales, convirtiéndolas en instituciones de larga duración.

En este sentido se entiende que la hegemonía en la producción de conocimiento, así como la formación y educación de las elites dirigentes, lo que ubicó a las universidades en un contexto de acciones diplomáticas hacia el exterior en una posición destacada dentro del sistema de instituciones. Por lo tanto, 
esta participación constante de las universidades las ubica como un nuevo actor en el sistema internacional capaz de ejercer diplomacia paralela a través de ciertas acciones como la internacionalización de la educación entre otras (Harispe, Herrero, \& Araya, 2014).

El posicionamiento a nivel local permite a las universidades mayor acceso a relaciones con otras, la cooperación internacional entre los centros universitarios no es un tema nuevo, desde la aparición de las tecnologías de la comunicación y las redes sociales.

Los actores subnacionales también se encuentran entre aquellos actores que buscan la internacionalización, entonces la definición de paradiplomacia abarca tanto a los municipios que forman parte del gobierno de un Estado-nacional, como también a otras instituciones, de significativo peso cultural y académico-científico. (Harispe, Herrero, \& Araya, 2014).

En los últimos años el mundo ha visto una creciente participación de los gobiernos regionales en el ámbito internacional, un fenómeno a veces conocido como paradiplomacia. Las razones radican tanto en cambios a nivel del sistema estatal e internacional, y en el plano político, así como el desarrollo económico dentro de las propias regiones.

\section{El ciudadano como protagonista de la paradiplomacia: Los programas de intercambio}

El término paradiplomacia universitaria resulta relevante en un contexto actual centralizado, entre reducción de presupuestos y la corrupción que impera en las instituciones, la relevancia del término recae en la estructura pura de la paradiplomacia entendida como las acciones diplomáticas llevadas a cabo por organismos no centrales en las relaciones internacionales a través del establecimiento de contactos ad hoc con entidades privadas o públicas del extranjero, con el fin de promover asuntos socioeconómicos y culturales, así como cualquier otra dimensión externa de sus competencias constitucionales (Cornago, 2000).

El papel del ciudadano en la paradiplomacia es primordial para aquellos que les preocupa su agenda de internacionalización pues el capital humano es esencial para que el proceso de internacionalización se lleve cabo exitosamente, de ahí la justificación del estudio de los estudiantes extranjeros de posgrado. En este sentido, Greogory menciona que su participación requiere una estrategia en donde estén involucrados ya sean actores estatales, sub-estatales o no estatales para formar una comprensión de las culturas, actitudes y comportamientos propios de una región o país, pues esto 
Zapata-Morán., M., G., Berlanga-Ramírez, J., H., \& Salazar-Mata, H.

conlleva a la construcción y gestión relaciones, para contribuir en el pensamiento de las audiencias y fomentar acciones que permitan favorecer sus intereses y valores (Greogory, 2011).

En este sentido los programas de intercambio capturan el interés de las élites académicas y crean conexiones de alto valor emocional, se le consideran estrellas de la paradiplomacia, pues permiten capturar la esencia de la sociedad civil a través del alumnado y sus docentes y experiencias en ambientes multiculturales, rodeados de libertad de pensamiento y conocimiento.

Cada alumno tiene la oportunidad de elegir el programa, la universidad o el destino. Se pretende que la experiencia sea única, aun cuando se enmarque en las acciones destinadas a colectivos académicos, periodísticos o de otra naturaleza (Manheim, 1994). Existen varios ejemplos de integración regional a través de las universidades, uno de ellos ha sido el programa Erasmus establecido por medio de la Unión Europea, que ha dado becas y financiamiento a jóvenes europeos que pretenden convertirse en ciudadanos globales y además contribuir con relaciones públicas a sus universidades.

Sin embargo, se encuentran algunas limitaciones para la medición de su desempeño pues son programas académicos de formación en el sentido amplio, pero también le consideran acciones que cuestan mucho en términos monetarios y de recurso humano y cuyo rendimiento es difícil de valorar.

El término paradiplomacia universitaria resulta relevante en un contexto actual centralizado, entre reducción de presupuestos y la corrupción que impera en las instituciones, la relevancia del término recae en la estructura pura de la paradiplomacia entendida como las acciones diplomáticas llevadas a cabo por organismos no centrales en las relaciones internacionales a través del establecimiento de contactos ad hoc con entidades privadas o públicas del extranjero, con el fin de promover asuntos socioeconómicos y culturales, así como cualquier otra dimensión externa de sus competencias constitucionales (Cornago, 2000).

Durante la crisis del COVID 19 se vislumbró que la situación de las instituciones de educación superior en cuanto su internacionalización sufriría una crisis sin precedentes, se tiene una oportunidad sin precedentes, ¿Qué se va a hacer con la internacionalización de la educación superior? ¿Cuáles serán las estrategias de paradiplomacia que impacten en el desarrollo institucional de las universidades? 


\section{El impacto del COVID-19 en la internacionalización de la universidad}

Recientemente, la internacionalización de la educación superior se ha visto profundamente afectada por las fuerzas gemelas de la pandemia de COVID -19 la política internacional. Si bien el resurgimiento del nacionalismo y la xenofobia en todo el mundo ya había arrojado dudas sobre la importancia de un sistema globalizado de educación superior, la pandemia solo se ha sumado al enigma al imponer restricciones al movimiento normal de personas dentro y entre las universidades del mundo.

A corto plazo, está claro que la pandemia ha afectado cambios masivos en los patrones que hemos llegado a observar en la educación superior internacional. El enorme experimento de la educación en línea nos dejará a todos permanentemente cambiados. Estaríamos mucho más dispuestos a participar en proyectos educativos a distancia. El trabajo que hemos estado haciendo en esta área durante los últimos años se consideraría preparatorio para un giro más generalizado hacia la enseñanza, la educación y la transferencia de conocimientos en línea. Las universidades se involucrarán más en este dominio que antes (Harvard, 2020, pp. 1).

Según el profesor Elliott (2020), podría ocurrir un posible aumento en el número de asociaciones entre universidades, y después del COVID-19, más personas en puestos administrativos podrán ver el valor de cultivar relaciones a nivel institucional. En este sentido, los acontecimientos del año 2020 han establecido la prioridad de invertir en salud, seguridad y la protección en el desarrollo, implementación y gestión de programas de intercambio internacional para la movilidad de estudiante y profesores.

Los líderes de la educación internacional comparten las lecciones aprendidas y ofrecen consejos prácticos sobre evaluaciones de riesgos, planes de emergencia y protocolos. ¿Cómo se pueden integrar las necesidades de salud, seguridad y protección de los programas de educación internacional en las relaciones con los socios y los planes estratégicos institucionales para garantizar un regreso seguro al estudiar en el extranjero?

\section{Estrategias de Paradiplomacia Universitaria}

En cuanto a la UANL es parte de 45 organizaciones mundiales con la intención de fortalecer el proceso de internacionalización y cuenta con tres oficinas en el extranjero para fomentar la paradiplomacia, sin embargo la importancia de la paradiplomacia y la cooperación entre actores a nivel internacional no se ha incorporado de manera definitiva en las agendas gubernamentales ni tampoco en las políticas educativas de los gobiernos estatales de México, debido a la carencia de diversos elementos tales como 
Zapata-Morán., M., G., Berlanga-Ramírez, J., H., \& Salazar-Mata, H.

recursos económicos, interés gubernamental, visión internacional y estrategia de vinculación internacional en materia de educación superior y en algunas ocasiones por la falta de compromiso de los lideres universitarios con la internacionalización, la poca información científica que se tiene al respecto, y modelos obsoletos de atención a estudiantes y profesores extranjeros (Waldman et al, 2018).

Existen estrategias que integran a la paradiplomacia, como lo son: La internacionalización de la planta docente, el financiamiento, los servicios de atención a estudiantes extranjeros, la proyección internacional, y los procesos de internacionalización que lleva cabo la universidad.

En este apartado se revisará el impacto que estas estrategias tienen en el ciudadano que interactúa con ellas de manera constante, en este caso el estudiante extranjero de posgrado de la Universidad Autónoma de Nuevo León.

Las universidades en México han estado en una búsqueda constante de estrategias que les permita formar parte del sistema internacional. Esto se puede observar en la cantidad de estudiantes o profesores extranjeros, y la cantidad de publicaciones con autores de otros países, los convenios vigentes, las redes científicas y académicas, asociaciones, entre otros.

La Universidad Autónoma de Nuevo León, se ha planteado estrategias y metas de internacionalización a través de sus planes de desarrollo institucional, en este sentido, En el año 2012, 597 estudiantes de la Universidad realizaron intercambio con otras instituciones: 27 con nacionales y 570 con internacionales, cifras que se vieron incrementadas para el 2018 con 853 estudiantes realizando intercambio hacia otras instituciones de educación superior: 69 nacionales y 784 internacionales. Mientras tanto, en el año 2012 la Universidad recibió a 301 estudiantes: 110 nacionales y 191 extranjeros, cantidad que también se vio incrementada en el 2018 con 700 estudiantes: 315 nacionales y 385 de otras partes del mundo (UANL, 2019).

Cuando se habla de movilidad de estudiantes, se entiende como aquellos que están estudiando fuera del lugar de origen. En un panorama internacional, el uso regular de los términos como estudiantes en el extranjero y académicos internacionales y flujos de estudiantes internacionales se han traducido a todos los idiomas e incluso han sido usados como sinónimos cuando son referidos (Altbach P., 1991).

De acuerdo con Gacel (2003) es necesario diseñar políticas de internacionalización que puedan contribuir a mejorar la calidad de la educación superior de manera que se puedan implementar cambios 
que transformen el sistema educativo que pueda enfrentar los nuevos retos de la globalización; sin embargo, el panorama para México no es del todo favorable pues la polaridad en la distribución de riquezas, la corrupción y la falta de inversión en la educación, necesitan la evaluación constante de la academia y diseñar estrategias de manera que los beneficios de la internacionalización a través de la cooperación puedan ser un beneficio que llegue a todos como una vía para mejorar los índices de competitividad en México (Imco, 2011).

Ahora bien, la internacionalización de la planta docente, la docencia son una parte fundamental de la paradiplomacia universitaria porque son parte de la movilidad académica y en otro porque son parte también de la calidad institucional en la internacionalización de la universidad a la que pertenecen. Los docentes y la educación de los docentes ocupan un lugar de privilegio en las agendas de las iniciativas internacionales, en los esfuerzos de la cooperación internacional y de los diseñadores de políticas nacionales. En muchos proyectos y programas dedicados a la reforma y el desarrollo educativos, las políticas o los estándares internacionales son producto de transferencias (Acedo, 2012).

El incremento de la movilidad de estudiantes y docentes, a nivel de grado y posgrado, es una meta deseable para los sistemas universitarios. Esta movilidad se constituye en un notable transmisor de la cooperación interinstitucional, sin embargo, debe de ir acompañado de mecanismos de redes académicas que se den a sí mismas resultados y objetivos de manera estructurada (Larrea \& Astur, 2017).

Las estrategias de financiamiento que lleve a cabo la universidad se consideran importantes para su proceso de internacionalización, por ejemplo, El tema de la globalización y la interculturalidad ha dado parte al análisis de distintos elementos, de los cuales, uno ha sido como es el contacto del estudiante extranjero con su universidad receptora. Por lo que hace algunos años las universidades receptoras como es el caso de la Universidad Nacional Autónoma de México y la UANL tuvieron que reorganizar su administración y establecer una oficina de administración de estudiantes extranjeros para satisfacer las necesidades únicas de los estudiantes extranjeros.

Los costos ejercen una influencia significativa en la elección de los estudiantes de una institución de educación superior. Soo y Elliot (2010) también estudiaron la influencia del costo financiero de asistir a una universidad por parte de estudiantes internacionales; sin embargo, sus estudios mostraron una relación no significativa entre las tarifas cobradas por una institución y la opción de inscribirse en la institución. La investigación también ha demostrado que la elección de las universidades por parte de los estudiantes está influenciada por los atributos de la universidad. 
Zapata-Morán., M., G., Berlanga-Ramírez, J., H., \& Salazar-Mata, H.

La reputación de una institución también es una consideración en la elección universitaria de un estudiante. Ming (2010) admite que la reputación de una institución es una poderosa influencia en los estudiantes potenciales. Dado el creciente número de instituciones de educación superior, los estudiantes son cada vez más críticos y analíticos en su selección de instituciones educativas.

Por otra parte, la globalización y la internacionalización de la educación superior han aumentado significativamente la competitividad en las universidades. La competencia entre universidades se observa de manera visible en los rankings universitarios. Estos rankings inevitables y probablemente necesarios clasifican a las mejores universidades según su calidad y prestigio (Carrillo \& Blanco, 2015).

La comunicación de la proyección internacional se debe de fundamentar en los mismos parámetros de gestión que la comunicación de la proyección internacional. Por otro lado, la comunicación online o en páginas web debe ofrecer a los públicos extranjeros la promesa que la universidad ofrece, reforzándola y aumentándola en distintos idiomas con los recursos que tenga a su alcance (Carrillo \& Blanco, 2015).

La gestión de la proyección internacional de las universidades, las acerca a los públicos extranjeros, pues es una manera de recibir la atención necesaria que les ayude a satisfacer sus necesidades. Implica la implementación de información web y la traducción de sus contenidos. Se requiere una participación mucho más activa para reforzar las actividades de las universidades.

Por otra parte, los servicios de atención de la escuela son una parte esencial de la calidad institucional que proyecte la universidad. La calidad institucional se entiende como el fortalecimiento de la capacidad de poner en práctica un sistema de calidad integral, efectivo y participativo para la ampliación de la oferta académica, los servicios administrativos y la planificación y la gestión (Miranda, 2004). Estudios anteriores demostraron el vínculo entre la calidad del servicio, la satisfacción de los estudiantes y la lealtad de los estudiantes, modelando las dinámicas existentes entre ellos (Teas \& Agarwal, 2000) y (Agarwal \& Teas, 2001). Dicho esto, la satisfacción de los estudiantes está doblemente vinculada a la calidad del servicio. En este contexto, las instituciones educativas deben monitorear activamente la calidad de los servicios que ofrecen y comprometerse con las mejoras continuas.

En este sentido Dorweiler y Yakhou citados por Petruzzellis (2010) mencionan que los estudiantes se han vuelto más discriminatorios en su selección y más exigentes con las universidades que 
eligen. Es importante, entonces, que las instituciones entiendan lo que los estudiantes desean y esperan de la institución que eligieron.

El servicio de atención que los estudiantes experimentan, su burocracia y su nivel de complejidad afectan en gran medida la primera impresión del servicio y la decisión inicial de solicitar un curso, así como el nivel de satisfacción del estudiante.

Un ejemplo de esto es, que casi dos tercios de los estudiantes de doctorado de los Estados Unidos aspiran a un puesto en un colegio o universidad. Aunque los estudiantes extranjeros esperan trabajar en un entorno académico, también aspiran a obtener relaciones duraderas con la universidad que los recibe a través de convenios con sus universidades de origen o redes académicas y de investigación internacionales (Altbach \& Knight, 2006)

\section{3.-MÉTODO}

\section{Diseño}

El enfoque cuantitativo de la investigación pretende confirmar la relación de las variables propuestas en la primera fase del estudio cualitativo a través de la generación de una encuesta compuesta de 43 reactivos en el cual se buscó investigar las orientaciones cognoscitivas, afectivas y evaluativas de los estudiantes que se tenga como muestra, con el objetivo de medir las percepciones y sentimientos que tienen los estudiantes hacia las acciones paradiplomáticas de su universidad receptora, en este caso la UANL.

\section{Participantes}

De 88 estudiantes de posgrado que se encuentran actualmente inscritos en la Universidad Autónoma de Nuevo León, se seleccionó una muestra de 72 estudiantes extranjeros, los cuales se segmentarán por zona a la que pertenece su país (América del Norte, Sudamérica, Europa, África y Asia) y por la rama del conocimiento al que pertenece su programa (Físico-matemáticas y ciencias de la tierra, Biología y química, Medicina y salud Humanidades, Sociales y Económicas, Biotecnología y agropecuarias e Ingeniería).

\section{Instrumentos}

Se operacionalizaron las variables, las dimensiones y los ítems, se establecieron los valores de la escala tipo Likert del 1 como Poco importante y 5 Muy importante. Después de revisarlo detenidamente, el 
Zapata-Morán., M., G., Berlanga-Ramírez, J., H., \& Salazar-Mata, H.

instrumento se aplicó a una muestra no probabilística de 15 estudiantes provenientes de Sudamérica, Europa y África que se inscribieron a programas de posgrado en la Universidad Autónoma de Nuevo León, en el periodo abril a mayo del año 2019. El instrumento está compuesto de 43 reactivos, los cuales fueron respondidos a través de correo electrónico y personalmente. Se realizó una prueba de fiabilidad, con la cual se trataba de comprobar que el instrumento es confiable, la confiabilidad demostrada en los resultados indica que el instrumento es aceptable y puede ser utilizado para recopilación de datos. Se procedió a aplicar la encuesta a la muestra correspondiente en el periodo de febrero a octubre del año 2020.

\section{Procedimientos}

En una primera fase del estudio se realizó una recopilación de documentos y literatura que proporcionara una visión integral de las variables de estudio, después se realizo el instrumento cuantitativo de encuesta que se aplicó a 72 estudiantes provenientes de otro país que estudian un posgrado de la UANL. Se hizo un análisis de fiabilidad para proceder a la interpretación de estadísticos descriptivos proporcionados por el software SPSS, con los cuales se pretende comprender el orden de importancia de los ítems del instrumento. Finalmente se redactaron resultados y conclusiones.

\section{4.-RESULTADOS}

\section{Análisis de fiabilidad}

El tiempo y el costo de postularse a la escuela de posgrado es alto, por lo que los posibles solicitantes y afiliados deben limitar sus opciones. Además, se están comprometiendo un mínimo de un año por lo que se invierte ese tiempo, fondos y esfuerzo sustancial, una vez que se comprometen a un solo programa de posgrado. Por lo tanto, la elección de a cuál asistir debe ser considerada cuidadosamente y las opciones sopesadas (Joseph, 2010).

El factor de interés en la investigación actual, en las escuelas de posgrado y para la universidad por igual, es comprender mejor cómo los estudiantes de posgrado llegan a su institución final de elección. Se compila y examina una lista de criterios de selección para programas de posgrado. En este sentido se encontró que la mayoría de los estudiantes, independientemente de su ciudadanía, tenían un objetivo a largo plazo de enseñar o un puesto de investigación en un entorno académico. 
Se elaboró el instrumento de medición, se optó por la elaboración de una encuesta con escala tipo Likert, que de acuerdo Bertram (2008) las escalas llamadas Likert son una escala de respuesta psicométrica utilizada principalmente en los cuestionarios para obtener preferencias o grado de acuerdo con una declaración o conjunto de declaraciones. Las escalas de Likert son una técnica de escala no comparativa y son unidimensionales (solo miden un único rasgo) en naturaleza. Se les pide a los encuestados que indiquen su nivel de acuerdo con una declaración dada a través de una escala ordinal.

"Únicamente, este tipo de instrumentos consten en una colección de items, la mitad manifestándose en una posición de acuerdo con la actitud a medir y la otra mitad en contra. Cada ítem está relacionado en una escala de valoración ordinal. Esta misma escala incorpora el punto medio neutral, así como los puntos a izquierda y derecha, originalmente de desacuerdo y de acuerdo, con opciones de respuesta numérica de 1 a 5. Esta escala de variables aparecía en horizontal, uniformemente espaciadas, al lado del ítem e incluía las etiquetas numéricas" (Matas, 2016, pp. 40).

Tabla 2.

Estrategias que impactan en la atracción de estudiantes extranjeros de la UANL Variable Media $\quad$ DE. Alfa de

\begin{tabular}{lccc} 
& Media & DE. & Alfa de \\
& & & Cronbach \\
\hline Planta docente & 3.02 & 1.22 & .793 \\
Oferta de Internacionalización & 3.07 & 1.36 & .909 \\
Financiamiento & 2.03 & 1.24 & .762 \\
Proyección internacional & 3.67 & .990 & .902 \\
Servicios de atención a estudiantes extranjeros & 3.53 & 1.10 & .809
\end{tabular}

Fuente: Elaboración propia, (2019).

Consecuentemente se procedió a realizar el análisis descriptivo de la muestra para la determinación del grado de relevancia de las variables y sus ítems. La desviación estándar es la forma de dispersión más común, que señala que tan diversos están los datos en relación con la media. Mientras mayor sea la desviación estándar, mayor será la dispersión de los datos. A continuación, se muestra el análisis por variable y el orden de relevancia para los entrevistados, lo que determinara el grado de importancia de cada uno de los ítems. 
Zapata-Morán., M., G., Berlanga-Ramírez, J., H., \& Salazar-Mata, H.

En primera instancia en la variable de planta docente los estudiantes extranjeros de posgrado determinaron que el ítem con mayor relevancia es el conocimiento multicultural de los profesores $(\mathrm{ME}=4.37, \mathrm{DE}=1,018)$, después impacto internacional de la producción académica de los profesores $(\mathrm{ME}=4.37, \mathrm{DE}=.914)$, posteriormente en cuanto a relevancia la variable de participación de los profesores en redes académicas internacionales( $\mathrm{DE}=4.32 \mathrm{ME}=.938)$ resultó ser importante, mientras que las variables accesibilidad de los profesores $(\mathrm{DE}=4.25 \mathrm{ME}=1.118)$ y Profesores especializados con algún título extranjero( $\mathrm{ME}=4.07, \mathrm{DE}=1.113)$ fueron las de menor importancia .

\section{Importancia Oferta de internacionalización}

En cuanto a la variable de oferta de internacionalización fueron generados indicadores que permitieran al estudiante medir en la toma de decisión la importancia de las estrategias de internacionalización de su escuela. En este sentido el ítem con mayor relevancia para los encuestados fue el de reconocimiento de la calidad educativa $(\mathrm{ME}=4.79, \mathrm{DE}=.558)$. También se observa que las alianzas internacionales de la universidad $(\mathrm{ME}=4.59, \mathrm{DE}=.599)$ resultó importante en el momento de tomar la decisión de asistir a una universidad; posteriormente el ítem reconocimiento de la universidad por evaluaciones internacionales $(\mathrm{ME}=4.56, \mathrm{DE}=.712)$. Sitio web de la universidad $(\mathrm{ME}=4.49, \mathrm{DE}=.791)$.

\section{Tabla 3.}

Descriptivos. Importancias de las variables oferta de internacionalización

\begin{tabular}{|c|c|c|c|c|c|}
\hline & N. & Mín. & Máx. & Media. & DE. \\
\hline OI 1. Reconocimiento de la calidad educativa & 71 & 2 & 5 & 4,79 & ,558 \\
\hline $\begin{array}{l}\text { OI 2. Oportunidades de colaborar en } \\
\text { investigaciones internacionales }\end{array}$ & 71 & 2 & 5 & 4,46 & ,842 \\
\hline $\begin{array}{l}\text { OI 3. Reconocimiento de la universidad por } \\
\text { evaluaciones internacionales }\end{array}$ & 71 & 2 & 5 & 4,56 & ,712 \\
\hline $\begin{array}{l}\text { OI 4. Las alianzas internacionales de la } \\
\text { universidad }\end{array}$ & 71 & 3 & 5 & 4,59 &, 599 \\
\hline $\begin{array}{l}\text { OI 5. Los convenios de cooperación de la } \\
\text { universidad con organismos internacionales }\end{array}$ & 71 & 2 & 5 & 4,48 & ,753 \\
\hline $\begin{array}{l}\text { OI 6. Oferta de redes de investigación } \\
\text { internacionales }\end{array}$ & 71 & 2 & 5 & 4,35 &, 864 \\
\hline $\begin{array}{l}\text { OI 7. La posibilidad de tener acceso a oficinas en } \\
\text { el extranjero de la universidad }\end{array}$ & 71 & 1 & 5 & 4,04 & 1,114 \\
\hline OI 8. Sitio web de la universidad & 71 & 2 & 5 & 4,49 & ,791 \\
\hline $\begin{array}{l}\text { OI 9. Posibilidad de llevar cursos o actividades en } \\
\text { plataforma digital }\end{array}$ & 71 & 1 & 5 & 3,85 & 1,359 \\
\hline OI 10. Disponibilidad de solicitud en línea & 71 & 1 & 5 & 4,28 & 1,003 \\
\hline $\mathrm{N}$ válido (según lista) & 71 & & & & \\
\hline
\end{tabular}


Fuente: Elaboración propia. (2020). Nota: Todas las variables independientes se midieron con escala tipo Likert con un rango teórico de variación de 1 (nada importante) a 5 (muy importante).

Los convenios de cooperación de la universidad con organismos internacionales $(\mathrm{ME}=4.48, \mathrm{DE}=$ .753); en grado de importancia sigue el ítem de oportunidades de colaborar en investigaciones internacionales $(\mathrm{ME}=4.46, \mathrm{DE}=.842)$; seguido de oferta de redes de investigación internacionales $(\mathrm{ME}=4.35, \mathrm{DE}=.864)$. Después el ítem disponibilidad de solicitud en línea $(\mathrm{ME}=4.28, \mathrm{DE}=1.003)$, y al final los ítems de la posibilidad de tener acceso a oficinas en el extranjero de la universidad (ME=4.04, $\mathrm{DE}=1.114)$, posibilidad de llevar cursos o actividades en plataforma digital $(\mathrm{ME}=3.85, \mathrm{DE}=1.359)$ que siguen estando por arriba de la media teórica.

Respecto a la variable de financiamiento el ítem de mayor importancia para los estudiantes extranjeros de posgrado fue el de asistencia financiera $(\mathrm{ME}=4.75, \mathrm{DE}=.691)$; seguido por el costo de colegiatura $(\mathrm{ME}=4.63, \mathrm{DE}=.882)$. Por otro lado, los ítems costo de la renta de vivienda $(\mathrm{ME}=4.39, \mathrm{DE}=$ 1.049) y costo del transporte $(\mathrm{ME}=4.17, \mathrm{DE}=1.207)$, también resultaron de importancia para los encuestados. El siguiente ítem medido en grado de importancia fue la disponibilidad de alojamiento seguro $(\mathrm{ME}=4.11, \mathrm{DE}=1.337)$, seguido por oportunidades de trabajar durante el curso $(\mathrm{ME}=3.52, \mathrm{DE}=$ 1. 611), y en menor grado de importancia fue la disponibilidad de beca en país de origen $(\mathrm{ME}=3.39$, $\mathrm{DE}=1.816)$.

Tabla 4.

Estadísticos descriptivos. Importancia de la variable Financiamiento

N. Mín. Máx. Media. DE.

\begin{tabular}{llllll}
\hline FN 1. Asistencia financiera (becas) país receptor (México) & 71 & 1 & 5 & 4,75 &, 691 \\
FN 2. Costo de colegiatura & 71 & 1 & 5 & 4,63 &, 882 \\
FN 3. Oportunidad de trabajar durante el curso. & 71 & 1 & 5 & 3,52 & 1,611 \\
FN 4. Disponibilidad de alojamiento seguro & 71 & 1 & 5 & 4,11 & 1,337 \\
FN 5. Costo de la renta de vivienda. & 71 & 1 & 5 & 4,39 & 1,049 \\
FN 6. Costo del transporte & 71 & 1 & 5 & 4,17 & 1,207 \\
FN 7. Disponibilidad de beca / ayuda fina & 71 & 1 & 5 & 3,39 & 1,816 \\
N válido (según lista) & 71 & & & &
\end{tabular}

Fuente: Elaboración propia. (2020). Nota: Todas las variables independientes se midieron con escala tipo Likert con un rango teórico de variación de 1 (nada importante) a 5 (muy importante). 
Zapata-Morán., M., G., Berlanga-Ramírez, J., H., \& Salazar-Mata, H.

La variable proyección internacional indicó que el ítem con mayor relevancia para los estudiantes extranjeros era la reputación de la escuela o facultad a nivel internacional ( $\mathrm{ME}=4.55 \mathrm{DE}=.628)$, seguido por la reputación de la universidad a nivel internacional $(\mathrm{ME}=4,51, \mathrm{DE}=.734)$, señalando que es importante la promoción de escuelas o facultades incluso en un mismo grado de importancia que el nombre de la universidad; después le sigue, promoción en congresos internacionales $(\mathrm{ME}=4,39 \mathrm{DE}=$ .819), participación en ferias internacionales $(\mathrm{ME}=4.31, \mathrm{DE}=.838)$ como factor de importancia en la selección de los estudiantes extranjeros de posgrado. Le siguen en importancia los ítems la página web de la universidad $(\mathrm{ME}=4.15, \mathrm{DE}=1$. 051), promoción en redes sociales $(\mathrm{ME}=3.86, \mathrm{DE}=1.175)$, Los ítems de menor importancia para los entrevistados fueron acceso a las páginas web en diferentes idiomas $(\mathrm{ME}=3.79, \mathrm{DE}=1.182)$, y marketing de los programas en páginas web $(\mathrm{ME}=3.73, \mathrm{DE}=1.133)$.

Tabla 5.

Estadísticos descriptivos. Importancia Variable Proyección Internacional

\begin{tabular}{lccccc}
\hline & N. & Mín. & Máx. & Media. & DE. \\
\hline $\begin{array}{l}\text { PI 1. Reputación de la Universidad/Facultad a nivel } \\
\text { internacional }\end{array}$ & 71 & 2 & 5 & 4,51 &, 734 \\
PI 2. Reputación de la escuela o facultad a nivel & 71 & 3 & 5 & 4,55 &, 628 \\
$\begin{array}{l}\text { internacional } \\
\text { PI. 3 participación en ferias internacionales }\end{array}$ & 71 & 2 & 5 & 4,31 &, 838 \\
PI 4. Promoción en congresos internacionales & 71 & 2 & 5 & 4,39 &, 819 \\
PI 5. Promoción en redes sociales & 71 & 1 & 5 & 3,86 & 1,175 \\
PI 6. La página web de la universidad & 71 & 1 & 5 & 4,15 & 1,051 \\
PI 7. Marketing de los programas en páginas web & 71 & 1 & 5 & 3,73 & 1,133 \\
PI 8. Acceso a las páginas web en diferentes idiomas & 71 & 1 & 5 & 3,79 & 1,182 \\
N válido (según lista) & 71 & & & & \\
\hline
\end{tabular}

Fuente: Elaboración propia. (2020). Nota: Todas las variables independientes se midieron con escala tipo Likert con un rango teórico de variación de 1 (nada importante) a 5 (muy importante).

Uno de los servicios que les pareció más relevante fue la recepción de estudiantes a nivel internacional $(\mathrm{ME}=4.69, \mathrm{DE}=.689)$, la amabilidad de los servicios escolares de admisión $(\mathrm{ME}=4.55$, $\mathrm{DE}=.842)$ y el Tiempo de respuesta de servicios escolares $(\mathrm{ME}=4.35, \mathrm{DE}=1,016)$; por otro lado, son importantes también las oportunidades de gestionar o colaborar en proyectos internacionales $(\mathrm{ME}=4.35$, $\mathrm{DE}=.927)$, y un proceso de admisión simple $(\mathrm{ME}=4.30, \mathrm{DE}=1.006)$. En último lugar de importancia 
resultan programas impartidos en idioma extranjero $(\mathrm{ME}=3.76, \mathrm{DE}=1.292)$, esto se podría deber a que la gran mayoría de los encuestados son de países de habla hispana.

\section{Tabla 6.}

Estadísticos descriptivos. Importancia Variable Servicios de Atención

\begin{tabular}{lccccc}
\hline & N. & Mín. & Máx. & Media & DE. \\
\hline SE 1. Un proceso de admisión simple & 71 & 1 & 5 & 4,30 & 1,006 \\
SE 2. Tiempo de respuesta de Servicios escolares de admisión & 71 & 1 & 5 & 4,35 & 1,016 \\
SE 3. Amabilidad de los servicios escolares de admisión & 71 & 1 & 5 & 4,55 &, 842 \\
SE 4. Oportunidades de gestionar o colaborar en proyectos & 71 & 1 & 5 & 4,35 &, 927 \\
internacionales & 71 & 2 & 5 & 4,69 &, 689 \\
SE 5. Recepción de estudiantes a nivel internacional & 71 & 1 & 5 & 3,76 & 1,292 \\
SE 6. Programas impartidos en idioma extranjero & 71 & & & & \\
N válido (según lista) & & & & & \\
\hline
\end{tabular}

Fuente: Elaboración propia. (2020). Nota: Todas las variables independientes se midieron con escala tipo Likert con un rango teórico de variación de 1 (nada importante) a 5 (muy importante).

Por último, en cuanto a la variable dependiente de intención de compra (intención de asistir a la UANL), el ítem de mayor relevancia fue: Yo planifiqué conscientemente asistir a mi universidad destino $(\mathrm{M}=4.41, \mathrm{DE}=.855)$ y las probabilidades de que yo eligiera mi universidad destino eran altas $(\mathrm{M}=4.27$, $\mathrm{DE}=1.203$ ) indicando la importancia de la intención de tomar la decisión de asistir a una universidad (destino). En menor grado de importancia se encuentran los ítems, mi primera opción de destino fue la UANL $(M=4.20, D E=1.203)$ y mi primera opción de universidad destino fue la universidad donde me encuentro actualmente $(M=4.14, \mathrm{DE}=1.175)$ indicando las distintas opciones que tienen los estudiantes en variedad de universidades con financiamiento.

\section{Tabla 7}

Estadísticos descriptivos. Importancia Variable Intención de Asistir a la UANL

\begin{tabular}{|c|c|c|c|c|c|}
\hline & N. & Mín. & Máx. & Media & DE. \\
\hline AS 1. Yo planifiqué conscientemente asistir a mi universidad destino & 71 & 1 & 5 & 4,41 & 855 \\
\hline $\begin{array}{l}\text { AS 2. Mi primera opción de universidad destino fue la universidad donde me } \\
\text { encuentro actualmente. }\end{array}$ & 71 & 1 & 5 & 4,14 & 1,175 \\
\hline AS 3. Las probabilidades de que yo eligiera mi universidad destino eran altas & 71 & 1 & 5 & 4,27 & 1,014 \\
\hline AS 4. Mi primera opción de destino fue la UANL & 71 & 1 & 5 & 4,20 & 1,203 \\
\hline $\mathrm{N}$ válido (según lista) & 71 & & & & \\
\hline
\end{tabular}

Fuente: Elaboración propia, (2020). Nota: Todas las variables independientes se midieron con escala tipo Likert con un rango teórico de variación de 1 (nada importante) a 5 (muy importante).

\section{5.- CONCLUSIONES}


Zapata-Morán., M., G., Berlanga-Ramírez, J., H., \& Salazar-Mata, H.

Todo esto se debe a la separación que se ha tenido de los gobiernos, el sector productivo y las universidades, además de la reducción de presupuestos a la ciencia, la cultura y la tecnología; si bien el CONACYT como parte angular de la internacionalización de las universidades, ha tenido algunas reestructuras durante los últimos años, fue palpable el limitado presupuesto para la ciencia, la educación y la tecnología en los últimos años comenzando desde el sexenio del presidente Enrique Peña Nieto y más reciente y notorio durante la pandemia, donde se veían casos de retrasos en pagos y paros de múltiples facultades, como es el caso de la UNAM que mantiene uno de los estándares más altos de relaciones y convenios con otras universidades o centros educativos y de investigación, así como redes de maestros y estudiantes globales.

Otro ítem relevante en el estudio "como supieron por primera vez de la universidad", un 54, 5\% contesto que fue a través de familiares o amigos, un $34.5 \%$ por Internet, $9.1 \%$ otro y $1.8 \%$ por redes sociales. Por lo que resulta de importancia la calidad del trato al estudiantes pues la comunicación de voz en voz de las experiencias vividas por los estudiantes (Word-of-Mouth Marketing) en el caso de la UANL es la principal forma de enterarse de la universidad. Seguido por el uso de internet por lo que se sugiere implementar uso de Big data, CRM, redes sociales más atractivas y páginas web más amigables para los estudiantes para una mayor interacción.

En la variable proyección internacional, el ítem con mayor relevancia para los estudiantes extranjeros fue la reputación de la escuela o facultad a nivel internacional, seguido por la reputación de la universidad a nivel internacional, en el aspecto en que se debe trabajar más en la defensa constitucional de la universidad pública mexicana para poder acceder a una universidad multicultural y con intercambios académicos más significativos, así como el acceso de las facultades a más convenios macros y ferias universitarias internacionales, pues en cuanto a los encuestados se refiere es de mayor importancia la imagen de la facultad como es el caso de escuelas.

Las estrategias de paradiplomacia medidas se sustentan en el que hacer institucional de la universidad, y las competencias que le permiten generarlas, en cuanto al impacto del COVID 19 se pudo encontrar un contraste en cuanto a la impartición de clases en línea y programas en idioma extranjero pues el instrumento se aplicó antes y durante la pandemia, permitiendo una medición de datos que resulta relevante en temporalidad. 
Al comienzo de la crisis, estuvo en el supuesto que las realidades en la educación superior internacional de antes de la crisis probablemente se mantendrían, y que la educación superior volvería rápidamente a las rutinas normales, pero tal vez con menos estabilidad financiera que antes. En muchos países e instituciones. Con la crisis en evolución y más países y personas afectadas, esa opinión ya no puede sostenerse.

En este contexto, se destaca la importancia de que los líderes de las escuelas de educación superior integren estrategias que permitan a las universidades contar con evaluaciones de riesgos, planes de emergencias y protocolos. En este sentido, las universidades podrían adaptar sus programas de educación internacional con estrategias paradiplomáticas con sus pares en el extranjero.

\section{REFERENCIAS}

Abdolalizadeh, M. (2014). Influential factor in international students' college choice of a rural, regional university. Kentucky: ProQuest LLC.

Acedo, C. (2012). Internacionalización de la educación docente. Perspectivas 42(1), 1-4.

Altbach, P. G. (1991). Impact and adjustment: foreign students in comparative perspective. Higher Education, 21 (3), 305-323.

Altbach , P. y Knight , J. (2006). Visión panorámica de la internacionalización en la educación superior: motivaciones y realidades. Perfiles educativos,28(112), 13-39.

Altbach, P. G. y De wit, H. (2020). El impacto del coronavirus en la educación superior. Nexos , 1-10 https://educacion.nexos.com.mx/?p=2221.

Amador, G. (2013). Política educativa y su relación con la internacionalización y la cooperación internacional en la educación superior: el caso de México. PORTES, Revista Mexicana de Estudios sobre la Cuenca del Pacífico, 13(7), 35-55.

Bertram, D. (2008). Likert Scales. Topic Report, 1-10.

Carrillo, V. y Blanco, T. (2015). La proyección internacional de las universidades españolas en sus sedes web. Universidad de Zulia, 183-203.

Cornago, N. (2000). Diplomacia, paradiplomacia redefinición de la seguridad mundial. Madrid: Marcial Pons.

Duchacek, I. (1986). The territorial dimension of politics: within, among and across nations. New York: Greenwood Press. 
Zapata-Morán., M., G., Berlanga-Ramírez, J., H., \& Salazar-Mata, H.

Elliot, M. (2021). What Is the Future of Higher Education's Internationalization? Hardvard Press, recuperado de: https://mittalsouthasiainstitute.harvard.edu/2020/08/future-of-higher-educationsinternationalization/

Farias, J. (2014). La paradiplomacia y la construcción de sus bases teóricas. CONfines 10(19), 159-164.

Gacel, J. (2003). La internacionalización de las universidades mexicanas. ANUIES 35(1) 133-142.

Greogory, B. (2011). American Public Diplomacy. The Hague Journal of Diplomacy 6, 351-372.

Harispe, A. y Araya, J. (2014). "Nuevos actores subnacionales en el sistema internacional: municipios y universidades". Ponencia presentada en el VII Congreso de Relaciones Internacionales del IRI, el I Congreso del CoFEI y el II Congreso de la FLAEI, Buenos Aires, Argentina, 26, 27 y 28 de noviembre de 2014.

Hull, D. (1986). Proceedings of the biennial meeting of the philosophy of science association. JSTOR.

Joseph, M. (2010). Graduate College Selection and its Impact on Branding: Journal of Academic Administration in Higher Education.

Larrea, M. y Astur, A. (2017). Políticas de internacionalización de la educación superior y cooperación internacional universitaria. UNICEN.

Matas, A. (2016). Diseño del formato de escalas tipo Likert: un estado de la cuestión. Redie, 38-47.

Melissen, J. (2005). The New Public Diplomacy: Soft Power and International. Basingstoke: Palgrave MacMillan.

Ming, J. (2010).Institutional factors influencing student's college choice decisions in Malaysia. Curtin University.1(3), 53-58.

ONU (2021). Ciudadanía global: una fuerza nueva y vital recuperado de https://www.un.org/es/chronicle/article/ciudadania-global-una-fuerza-nueva-y-vital

Petruzzellis, L. y Romanazzi, S. (2010). Educational value: how students choose university, Emerald 24(3), 139-158.

Ruiz-Gutierrez, C. (2014). Internacionalización de la educación superior, Paradiplomacia universitaria y la gestión de la comunicación en este proceso. Piura Peru: Universidad de Piura.

Sebastian, J. (2004). Cooperación e internacionalización de las universidades, 211. Recuperado de: http://www.iesalc.unesco.org.ve/index.php?option=com_content\&view=article\&id=242

Soldatos, P. (1990). An Explanatory Framework for the Study of Federated States as Foreign-Policy Actors. Units, Oxford University press 34-38. 
Teas, K. y Agarwal, S. (2000). The effects of extrinsic product cues on consumers perceptions of quality, sacrifice, and value. Journal of the Academy of Marketing Science, 3(4), 278-290.

UANL, U. (2020). Plan Institucional de Desarrollo 2030. UANL.

Velázquez, R. (2007). La Paradiplomacia mexicana: Las relaciones exteriores de las entidades mexicanas. México: CIDE.

García-Waldman, Jiménez y Zapata-Morán, (2019), La paradiplomacia universitaria: La internacionalización de la educación superior en América Latina, Revista Política, Globalidad y Ciudadanía DOI: http://dx.doi.org/10.29105/p4.8-3.

Zapata Moran, M. (2021). Las estrategias de Paradiplomacia Universitaria en la atracción de estudiantes extranjeros de Posgrado de la Universidad Autónoma de Nuevo León publicado en Universidad Autónoma de Nuevo León.

Zeraoui, Z. (2016). Para entender la Paradiplomacia. Desafíos. 